\title{
Modelling Nonlinear Ultrasound Propagation in Absorbing Media using the k-Wave Toolbox: Experimental Validation
}

\author{
Kejia Wang*, Emily Teoh*, Jiri Jaros ${ }^{\dagger}$, and Bradley E. Treeby* ${ }^{*}$ \\ ${ }^{*}$ Research School of Engineering, College of Engineering and Computer Science \\ ${ }^{\dagger}$ Research School of Computer Science, College of Engineering and Computer Science \\ The Australian National University, Canberra, ACT 0200, Australia \\ ‡Email: bradley.treeby@anu.edu.au
}

\begin{abstract}
The simulation of nonlinear ultrasound waves in biological tissue has a number of important applications. However, this is a computationally intensive task due to the large domain sizes required for many problems of practical interest. Recently, an efficient full-wave nonlinear ultrasound model was developed and released as part of the open source $k$-Wave Toolbox. Here, this model is validated using a series of experimental measurements made with a linear diagnostic ultrasound probe and a membrane hydrophone. Measurements were performed in both deionised water and olive oil, the latter exhibiting power law absorption characteristics similar to human tissue. Steering angles of $0^{\circ}$ and $20^{\circ}$ were also tested, with propagation distances on the order of hundreds of acoustic wavelengths. The simulated and experimental results show a close agreement in both the time and frequency domains. These results demonstrate the quantitative validity of performing nonlinear ultrasound simulations using the k-Wave toolbox.
\end{abstract}

\section{INTRODUCTION}

Accurately simulating the propagation of nonlinear ultrasound waves through soft biological tissue is important for a number of applications, including equipment design, dosimetry, and computer-aided diagnosis. Currently, most nonlinear ultrasound simulations are based on variations of the Khokhlov-Zabolotskaya-Kuznetsov (KZK) or Burgers equations [1], [2]. Whilst these models are accurate for many practical situations [3], they are restricted to modelling directional sound beams, and often only consider one-way wave propagation in homogeneous media [4]. Recently, a full-wave nonlinear ultrasound model based on the $k$-space pseudospectral method was developed and released as part of the open-source k-Wave Acoustics Toolbox [5], [6]. This model can account for the propagation of nonlinear ultrasound waves in generally heterogeneous media including power law acoustic absorption, with no restrictions on the directionality of the waves. The objective of the current work was to validate this model using experimental measurements of the ultrasound fields produced by a diagnostic ultrasound transducer. Comparisons were made with both on and off axis beam patterns and in absorbing fluids. This builds on earlier work to validate $\mathrm{k}$-Wave for modelling problems in photoacoustics when the wave propagation is assumed to be linear and lossless [7].

\section{METHODS}

\section{A. Experimental Measurements}

The experiments were performed in a $40 \times 40 \times 60 \mathrm{~cm}$ test tank using a two-axis computer controlled positioning system with an accuracy of $\pm 2.5 \mu \mathrm{m}$ (Precision Acoustics, Dorchester, UK). The two motorised axes were aligned in either the $x-y$ plane to acquire data perpendicular to the beam axis, or the $x-z$ plane to acquire data along the beam axis (see Fig. 1). In both cases, the third axis was controlled by a manual translation stage with a positioning accuracy of $\pm 50 \mu \mathrm{m}$. The positioning arm was used to hold a calibrated PVDF membrane hydrophone with a thickness of $15 \mu \mathrm{m}$ and a $0.4 \mathrm{~mm}$ active element (Precision Acoustics, Dorchester, UK). Time domain signals at each spatial position were automatically acquired using a digital storage oscilloscope with a $200 \mathrm{MHz}$ bandwidth controlled by the positioning software (DSO-X 3024A, Agilent Technologies, Santa Clara, $\mathrm{CA}$ ). The signals were acquired using a sampling frequency of $2 \mathrm{GHz}$ and 512 averages.

A SONIX RP diagnostic ultrasound scanner (Ultrasonix, British Columbia, Canada) with an L9-4/38 linear probe was used to generate the ultrasound waves. The probe had 128 rectangular elements with an element height of $6 \mathrm{~mm}$, element width of $0.2698 \mathrm{~mm}$, kerf width of $0.035 \mathrm{~mm}$, and a fixed elevation focus of $19 \mathrm{~mm}$. The transmit pulse was programmed using the Texo SDK with 32 active elements, a transmit frequency of $5 \mathrm{MHz}$, a pulse shape of ' $+-+-+-{ }^{\prime}$, and a transmit power of $12 \mathrm{~V}$. The transducer was enclosed in a plastic probe cover (Cone Instruments, Solon, $\mathrm{OH}$ ) with a thin covering of ultrasound gel, and held in the tank using a probe holder (see Fig. 1).

Two different experiments were performed. In the first experiment, time domain pressure signals in four planes perpendicular to the beam axis were acquired (the $x-y$ plane shown in Fig. 1). The planes were $10 \times 15 \mathrm{~mm}$ in size and spaced $10 \mathrm{~mm}$ apart in the $z$-direction, with the first plane $1.3 \mathrm{~mm}$ from the probe face (this distance was measured using the SONIX RP imaging software). The electronic transducer focus distance in the beam plane was set to $21.3 \mathrm{~mm}$ to 
coincide with the third measurement plane. Within each plane, $100 \times 150$ waveforms were acquired with a step size of $100 \mu \mathrm{m}$. The acquisition of each waveform and subsequent movement of the hydrophone (including a $250 \mathrm{~ms}$ settle time) took just under 1 second, with each measurement plane taking on the order of 4 hours to complete. The experiment was repeated using both deionised water and olive oil (ExtraVirgin Olive Oil, Olives Direct, Nambour, Australia) at room temperature $\left(22^{\circ} \mathrm{C} \pm 1^{\circ} \mathrm{C}\right)$. Olive oil was chosen as a test fluid as it exhibits power law acoustic absorption characteristics similar to those observed in human tissue (see Table I).

In the second experiment, time domain pressure signals within the beam plane were acquired. The ultrasound probe was rotated $90^{\circ}$ about the $z$-axis compared to the alignment shown in Fig. 1, and the hydrophone was moved through the $x-z$ plane. The $2 \mathrm{D}$ scan plane was aligned with the beam plane by progressively moving the hydrophone to the beam centre in the $y$-direction (using the manual translation stage) at two different $z$-positions $(5 \mathrm{~mm}$ and $30 \mathrm{~mm}$ from the transducer face). The experiment was performed in deionised water using the same transmit pulse as the first experiment, and repeated for electronic steering angles of $0^{\circ}$ and $20^{\circ}$. For a steering angle of $0^{\circ}$, the scan area was $10 \times 30 \mathrm{~mm}$, and for $20^{\circ}$ it was $20 \times 30 \mathrm{~mm}$. In both cases, the step size was $100 \mu \mathrm{m}$, and the electronic focus distance was $20 \mathrm{~mm}$.

\section{B. $k$-Wave Simulations}

The simulations were performed using the nonlinear ultrasound model included the k-Wave Toolbox as described in [5]. This model is based on the iterative solution of three coupled first-order partial differential equations which describe the propagation of nonlinear ultrasound waves in heterogeneous media with power law acoustic absorption. (These equations are equivalent to a generalised form of the Westervelt equation.) The complete spatial domain in $3 \mathrm{D}$ is discretised, and the solution for the pressure and particle velocity fields everywhere within the domain are calculated in a time-stepping fashion. This is different to one-way models (for example the KZK Texas code [1]) which propagate the complete time trace of the pressure signals between parallel planes. The governing equations in $\mathrm{k}$-Wave are solved using the $k$-space pseudospectral method [8]. Compared to finitedifference and finite-element methods, this allows for much coarser grid spacings and larger time steps for the same degree of accuracy [8], [5].

The experimental signals measured in the $x-y$ plane closest to the ultrasound probe were used as the input to the simulations. The signals were temporally down-sampled (using an anti-aliasing filter) by a factor of 10 to give a time step of $5 \mathrm{~ns}$, and spatially up-sampled by a factor of 4 using nearest neighbour interpolation to give a grid point spacing of $25 \mu \mathrm{m}$. These parameters were used to define the simulation grid, giving a maximum supported frequency of $30 \mathrm{MHz}$ and a Courant-Friedrichs-Lewy (CFL) number of 0.3 . The total grid size was $432 \times 640 \times 1250$ grid points $(10 \times 15 \times 30 \mathrm{~mm})$ including a perfectly matched layer (PML) of $16 \times 20 \times 25$

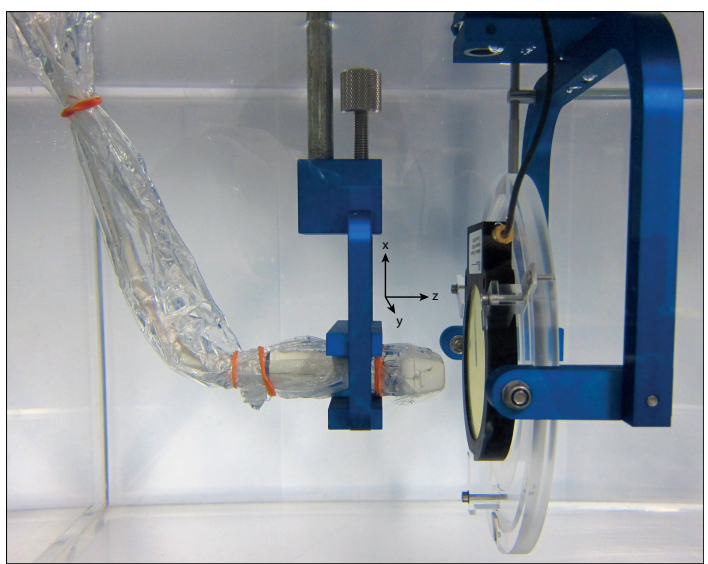

Fig. 1. Photograph of the ultrasound test tank showing the linear ultrasound probe, membrane hydrophone, and computer controlled positioning system.

TABLE I

ACOUSTIC PROPERTIES OF WATER AND OLIVE OIL USED FOR THE K-WAVE SIMULATIONS. VALUES ARE TAKEN FROM: $*[9]^{\dagger}[10]^{\ddagger}[11]^{\S}[12]$

\begin{tabular}{cccccc}
\hline & $\begin{array}{c}c_{0} \\
{[\mathrm{~m} / \mathrm{s}]}\end{array}$ & $\begin{array}{c}\rho \\
{\left[\mathrm{kg} / \mathrm{m}^{3}\right]}\end{array}$ & $B / A$ & $\begin{array}{c}\alpha_{0} \\
{\left[\mathrm{~dB} /\left(\mathrm{MHz}^{y} \mathrm{~cm}\right)\right]}\end{array}$ & $y$ \\
\hline water & $1482^{*}$ & $1000^{*}$ & $4.96^{*}$ & $2.17 \mathrm{e}-3^{*}$ & $2^{*}$ \\
olive oil & $1446^{\dagger}$ & $916^{\ddagger}$ & $11.1^{\S}$ & $0.13^{\dagger}$ & $1.68^{\dagger}$ \\
\hline
\end{tabular}

grid points on each side of the domain. The PML sizes were chosen to give grid dimensions with small prime factors to minimise compute times (the model makes heavy use of the FFT which is fastest for grid dimensions that are powers of two or have small prime factors).

The input pressure signals were assigned to source.p and enforced as a time-varying Dirichlet boundary condition over a $10 \times 15 \mathrm{~mm}$ plane within the computational grid by setting source.p_mode $=$ 'dirichlet'. The acoustic properties of the medium were defined to be homogeneous using the book values given in Table I. The sensor mask used to record the output from the simulations was defined to be the $x-y$ and $x-z$ planes matching the size and position of the scan planes used in the experiments.

The simulations were performed in single precision using $\mathrm{C}++$ versions of kspaceFirstorder3D written for shared memory and distributed memory platforms [5]. The shared memory simulations were run on a Tyan server with two six-core Intel Xeon X5650 processors [5]. The distributed memory simulations were run on the VAYU supercomputer (based on Sun X6275 blades) at the NCI national facility. Each simulation took between 2 hours (distributed memory code running on 256 cores) and 13 hours (shared memory code running on 12 cores), and used 23 GB of memory.

\section{RESULTS}

A comparison of the waveforms in the centre of each plane (i.e., along the beam axis) for the first experiment in deionised water is shown in Fig. 2(a). The left panels show the time domain pressure signals, and the right panels show the 
corresponding amplitude spectrums. The distances given are relative to the input plane, and the time domain signals have been aligned to correct for minor errors in the sound speed used in the simulations. The pressure is highest in the beam focus, and the wave steepens with distance due to cumulative nonlinear effects. There is a very close agreement between the experimental data and the simulated results in absolute units. No numerical dispersion is evident in the simulated results, even for the final measurement plane which is $30 \mathrm{~mm}$ from the input. This distance corresponds to 100 acoustic wavelengths at the transmit frequency, and 600 wavelengths at the highest modelled harmonic.

The equivalent results for the experiments in olive oil are shown in Fig. 2(b). For these experiments, the sensitivity of the hydrophone was significantly increased (using the standard calibration data, the pressure magnitudes measured in the plane closest to the ultrasound probe were nearly 3 times greater in olive oil compared to water for the same transmit settings). This change in sensitivity might be due to improved coupling between the hydrophone and the olive oil, or to viscous heating within the boundary layer between the oil and the membrane (further analysis is required). To account for this change in sensitivity, an additional calibration factor was introduced to normalise the maximum pressure measured in the plane closest to the probe in olive oil against the equivalent maximum pressure measured in deionised water.

There is again a good agreement between the experimental data and the simulated results in both the time and frequency domains. Compared to the measurements in deionised water, the increased acoustic absorption in olive oil results in a decrease in the pressure magnitudes. In particular, the higher frequency harmonics in the final measurement plane have been noticeably diminished. The slightly larger deviation between the results in the beam focus might be due to a weak thermal lensing effect caused by localised heating of the olive oil through acoustic absorption [13]. Other possible sources of error include uncertainties in the hydrophone calibration, uncertainties in the material properties, small alignment errors, and slight changes in temperature over the course of the experiment. This experiment provides a realistic test of the numerical model, as the acoustic absorption in olive oil is similar to that in soft biological tissue. The ability to directly and efficiently model acoustic absorption following a frequency power law is one of the strengths of the model used in k-Wave [14].

A comparison of the beam patterns recorded in the azimuth plane of the transducer is shown in Fig. 3. The total beam patterns were extracted using the integral of the amplitude spectrum at each spatial position, while the beam patterns at the fundamental and second harmonic correspond to the relative spectral amplitudes at 5 and $10 \mathrm{MHz}$. For a steering angle of $0^{\circ}$, there is a good agreement between the simulated and experimental results. In particular, the interference fringes at the fundamental frequency, and the spatial extent and magnitude of the second harmonic are closely matched. Similar results are obtained for a steering angle of $20^{\circ}$. In both cases, the small variations can be attributed to difficulties in aligning (a) Deionised Water
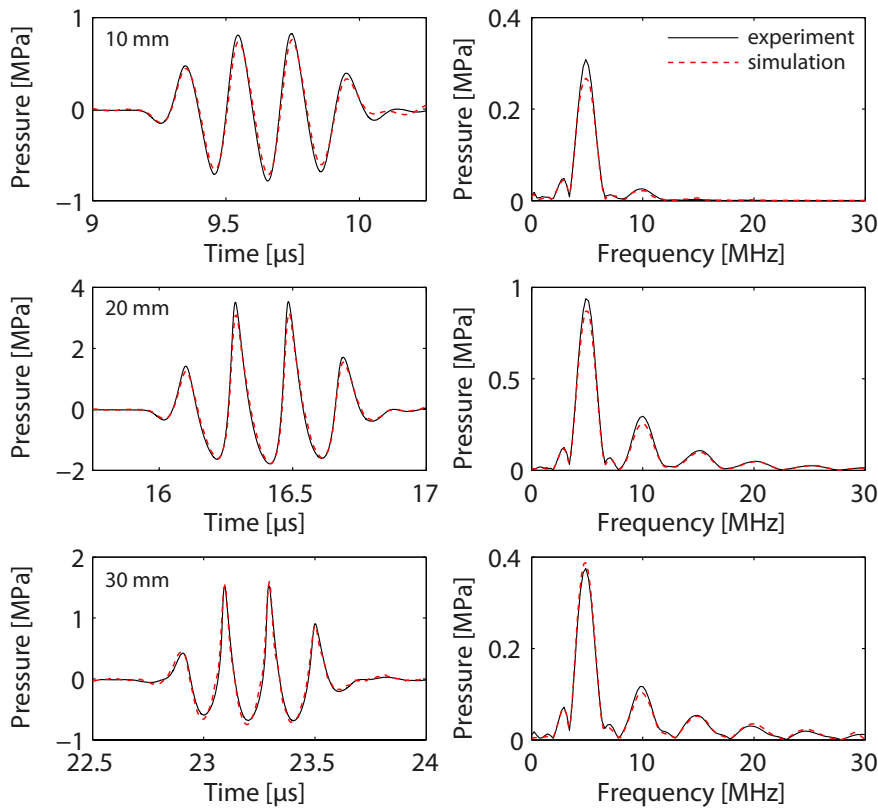

(b) Olive Oil
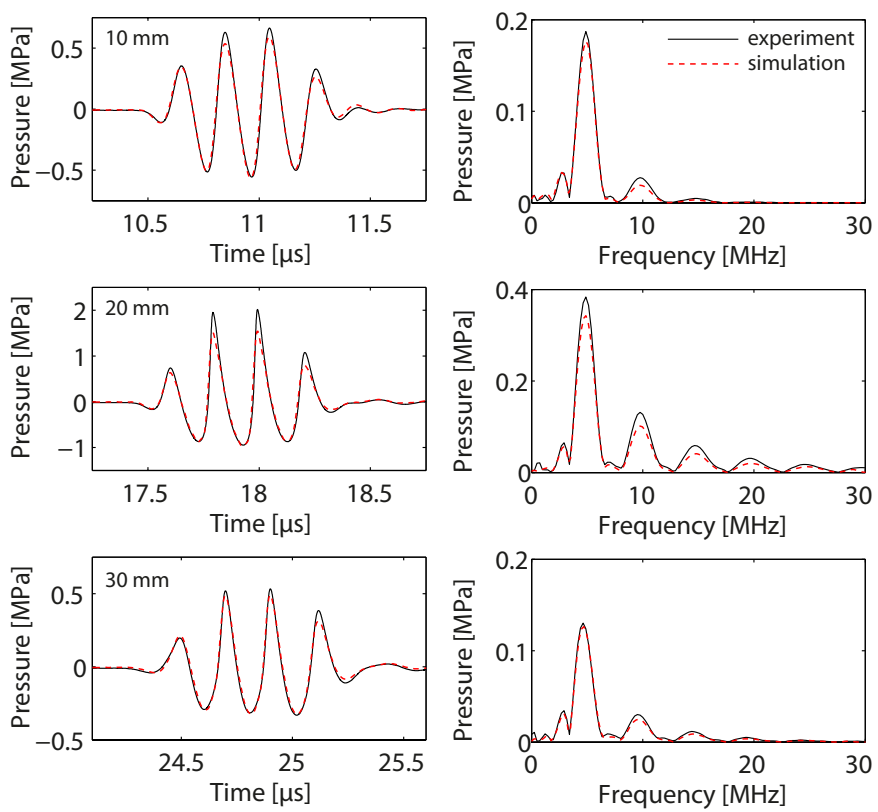

Fig. 2. Comparison of experimental (solid black line) and simulated (dashed red line) pressure signals along the axis of an ultrasound beam produced by a diagnostic ultrasound probe in (a) deionised water, and (b) olive oil at $22^{\circ} \mathrm{C}$.

the scan plane exactly through the centre of the beam plane. In the experiment, the scan plane was aligned by progressively moving to the centre of the beam at varying distances from the transducer face. However, this approach is unable to account for angular misalignment about the beam axis. In the simulation, the scan plan was chosen to be the Cartesian plane in the $x-z$ direction intersecting the beam focus. However, if the measured $x-y$ plane used for the simulation input is not exactly perpendicular to this, the results close to the transducer face will be misaligned. 


\section{CONCLUSION}

This paper reports the first series of experiments used to validate the accuracy of the nonlinear ultrasound model in the $\mathrm{k}$-Wave Toolbox. Experiments were performed in deionised water and olive oil using a linear ultrasound probe. Beam patterns for steering angles of $0^{\circ}$ and $20^{\circ}$ were also compared. There was a close agreement between the simulated and experimental results in both the time and frequency domains. These results demonstrate the quantitative validity of performing ultrasound simulations using the k-Wave toolbox. Future experiments will compare results for a wider range of transducers and steering angles, and for heterogeneous media.

\section{ACKNOWLEDGMENT}

This work was supported in part by the Australian Research Council/Microsoft Linkage Project LP100100588, and computational resources from the NCI National Facility at the Australian National University (ANU). KW is currently at the University of Auckland and was supported by a Summer Research Scholarship at the ANU. The authors would like to thank Ben Cox for useful discussion.

\section{REFERENCES}

[1] R. O. Cleveland, M. F. Hamilton, and D. T. Blackstock, "Time-domain modeling of finite-amplitude sound in relaxing fluids," J. Acoust. Soc. Am., vol. 99, no. 6, pp. 3312-3318, 1996.

[2] T. Varslot and G. Taraldsen, "Computer simulation of forward wave propagation in soft tissue," IEEE Trans. Ultrason. Ferroelectr. Freq. Control, vol. 52, no. 9, pp. 1473-1482, 2005.

[3] M. A. Averkiou and R. O. Cleveland, "Modeling of an electrohydraulic lithotripter with the KZK equation." J. Acoust. Soc. Am., vol. 106, no. 1, pp. 102-112, 1999.

[4] G. F. Pinton and G. E. Trahey, "A comparison of time-domain solutions for the full-wave equation and the parabolic wave equation for a diagnostic ultrasound transducer." IEEE Trans. Ultrason. Ferroelectr. Freq. Control, vol. 55, no. 3, pp. 730-733, 2008.

[5] B. E. Treeby, J. Jaros, A. P. Rendell, and B. T. Cox, "Modeling nonlinear ultrasound propagation in heterogeneous media with power law absorption using a k-space pseudospectral method," J. Acoust. Soc. Am., vol. 131, no. 6, pp. 4324-4336, 2012.

[6] B. E. Treeby and B. T. Cox, "k-Wave: MATLAB toolbox for the simulation and reconstruction of photoacoustic wave fields," J. Biomed. Opt., vol. 15, no. 2, p. 021314, 2010.

[7] B. T. Cox, J. Laufer, K. Kostli, and P. C. Beard, "Experimental validation of photoacoustic k-space propagation models," in Proc. SPIE, vol. 5320, 2004, pp. 238-248.

[8] M. Tabei, T. D. Mast, and R. C. Waag, "A k-space method for coupled first-order acoustic propagation equations," J. Acoust. Soc. Am., vol. 111, no. 1 , pp. 53-63, 2002.

[9] T. L. Szabo, Diagnostic Ultrasound Imaging. Burlington: Elsevier, 2004.

[10] B. E. Treeby, B. T. Cox, E. Z. Zhang, S. K. Patch, and P. C. Beard, "Measurement of broadband temperature-dependent ultrasonic attenuation and dispersion using photoacoustics," IEEE Trans. Ultrason. Ferroelectr. Freq. Control, vol. 56, no. 8, pp. 1666-1676, 2009.

[11] J. N. Coupland and D. J. McClements, "Physical properties of liquid edible oils," J. Am. Oil Chem. Soc., vol. 74, no. 12, pp. 1559-1564, 1997.

[12] E. C. Everbach and R. E. Apfel, "An interferometric technique for B/A measurement," J. Acoust. Soc. Am., vol. 98, no. 6, pp. 3428-34328, 1995.

[13] I. M. Hallaj, R. O. Cleveland, and K. Hynynen, "Simulations of the thermo-acoustic lens effect during focused ultrasound surgery," $J$. Acoust. Soc. Am., vol. 109, no. 5, pp. 2245-2253, 2001.

[14] B. E. Treeby and B. T. Cox, "Modeling power law absorption and dispersion for acoustic propagation using the fractional Laplacian," $J$. Acoust. Soc. Am., vol. 127, no. 5, pp. 2741-2748, 2010.
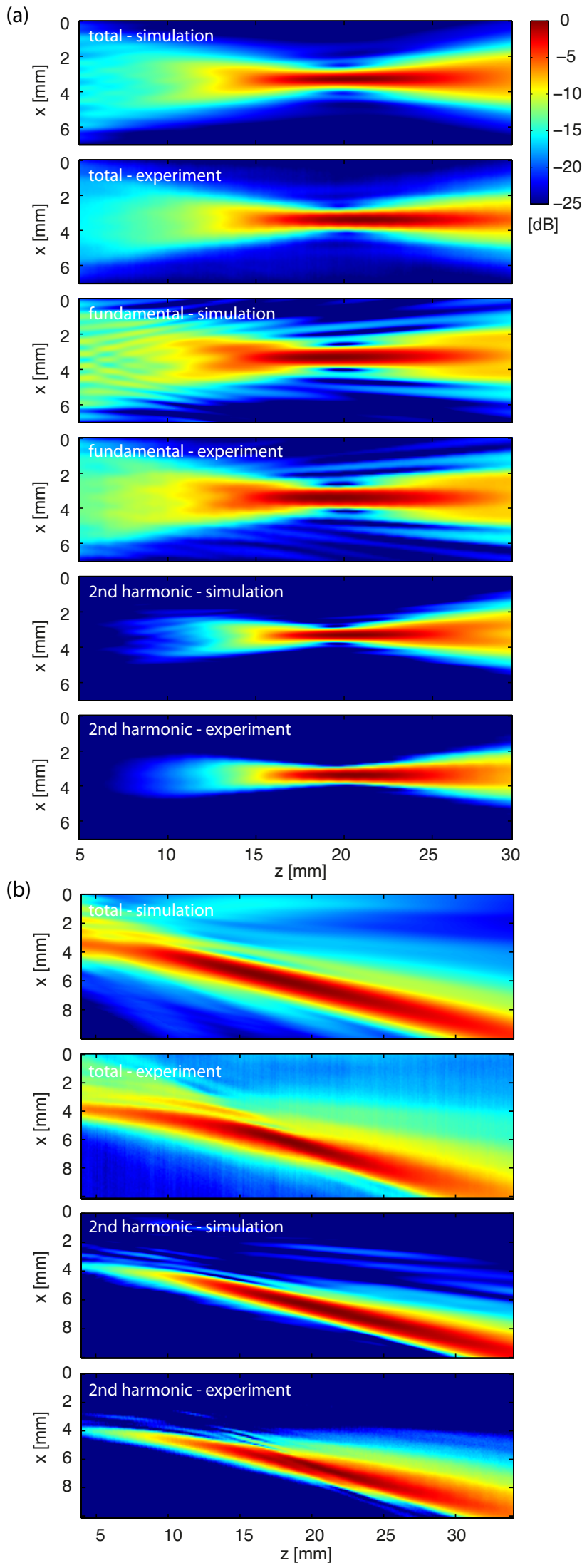

Fig. 3. Experimental and simulated beam patterns produced by a diagnostic ultrasound probe in deionised water for steering angles of (a) $0^{\circ}$ and (b) $20^{\circ}$. 